\title{
Clinical outcomes in elderly kidney transplant recipients: emphasis on choice of induction immunosuppressive therapy
}

\author{
Kyeong Deok Kim, Kyo Won Lee, Sang Jin Kim, Okjoo Lee, Jieun Kwon, Eun Sung Jeong, Jaehun Yang, Manuel Lim,
} Jae Berm Park

Division of Transplantation, Department of Surgery, Samsung Medical Center, Seoul, Korea

Background: Patients waiting for kidney transplantation (KT) and recipients who underwent KT over age of 60 years are increasing annually. The increased age of the recipients is known to be associated with decrease in the rejection rate. However, infections are more frequent in older recipients. The aim of this study was to evaluate the infection and rejection rate according to the recipients' age and induction agents.

Methods: All patients who underwent KT from June 2011 and April 2019 were retrospectively reviewed. The patients were divided into four groups according to their age ( $<60$ years and 60 years) and the type of induction agents used (basiliximab $20 \mathrm{mg}$ twice and rabbit anti-thymocyte globulin [r-ATG] $1.5 \mathrm{mg} / \mathrm{kg}$ for 3 days).

Results: The 704 recipients were included (basiliximab and young age group, $n=287$ [40.8\%]; basiliximab and old age group, $n=59$ [8.4\%]; r-ATG and young age group, $n=238$ [33.8\%]; basiliximab and old age group, $n=120$ [17.0\%]). The rejection rate within 1 year was similar between young and old age group regardless of the type of induction agents. Cytomegalovirus antigenemia, BK viremia, Pneumocystis carinii pneumonia, pneumonia, and blood stream infection rate within 1 year were similar between young and old age group in basiliximab $(P=0.200, P=0.244, P=0.312, P=0.248$, and $P=0.064$ respectively); however, they were significantly higher in the old age group in $r-A T G(P<0.001, P=0.036, P=0.037, P=0.049$ and $P=0.008$ respectively).

Conclusions: The rejection rate and most of the infections were not influenced by age when basiliximab was used as an induction agent. The rejection rate was not significantly different between the young and old age group; however, most of the infections were more common in old age group when r-ATG was used as an induction agent. Thus, dose reduction of r-ATG might be needed for elderly patients.

Corresponding author: Kyo Won Lee

E-mail: kw1980.lee@gmail.com

(C) The Korean Society for Transplantation

This is an Open Access article distributed under the terms of the Creative Commons Attribution Non-Commercial License (http://creativecommons.org/licenses/by-nc/4.0/) which permits unrestricted non-commercial use, distribution, and reproduction in any medium, provided the original work is properly cited. 